\title{
Iterative Detection of Multicode DS-CDMA Signals with Strongly Nonlinear Transmitters
}

\author{
Rui Dinis \\ Instituto de Telecomunicações/FCT-UNL \\ Av. Rovisco Pais, Torre Norte, Lisboa, Portugal \\ Email: rdinis@ fct.unl.pt
}

\author{
Paulo Silva \\ Instituto de Telecomunicações/ISE-Ualg. \\ Av. Rovisco Pais, Torre Norte, Lisboa, Portugal \\ Email:psilva@ualg.pt
}

\begin{abstract}
Whenever a DS-CDMA signal is the sum of several components associated to different spreading codes it has high envelope fluctuations and a high peak-to-mean envelope power ratio (PMEPR) setting strong linearity requirements for the power amplifiers. The use of clipping and filtering techniques (which can be repeated several times) was shown to be an effective way of reducing the PMEPR of DS-CDMA signals.

We consider an improved receiver where there is an iterative estimation and cancelation of nonlinear distortion effects. The proposed receiver allows significant performance improvements after just a few iterations, even when we have strong nonlinear distortion effects.
\end{abstract}

\section{INTRODUCTION}

The envelope fluctuations and peak-to-mean envelope power ratio (PMEPR) of DS-CDMA signals can be very high when we combine a large number of signals with different spreading codes, namely at the downlink transmission and/or for multicode CDMA schemes [1], leading to amplification difficulties. For this reason, several techniques were proposed for designing low-PMEPR DS-CDMA signals [2], [3].

A simple method to reduce the PMEPR of DS-CDMA signals it to employ clipping and filtering techniques [3]. However, the filtering operation produces some envelope fluctuations regrowth, limiting the achievable PMEPR [3]. By repeating the clipping and filtering $(\mathrm{C} \& \mathrm{~F})$ procedures we can reduce the PMEPR regrowth in multicode DS-CDMA schemes [4]. However, nonlinear distortion levels increase when we repeat the $\mathrm{C} \& \mathrm{~F}$ procedures, leading to performance degradation. This performance degradation can be particularly high when we have different powers assigned to different spreading codes, especially for the codes with lower power [5]. A scenario where this effect might be significant is for multi-resolution broadcasting systems [6], where we transmit simultaneously several parallel data streams with different powers so as to have different error protections. For DS-CDMA systems, this can be achieved by assigning to each resolution a subset of the available spreading codes and a different power to each subset (i.e., the spreading codes with higher power have higher error protection and, therefore, are associated to the basic (lower) resolution). We can improve significantly the

\footnotetext{
${ }^{1}$ This work was partially supported by Fundação para a Ciência e Tecnologia (pluriannual funding, U-BOAT project PTDC/EEA-TEL/67066/2006 and the FCT/POCI 2010 research grant SFRH / BD / 24520 / 2005) and the European IST project C-MOBILE.
}

performance of nonlinearly-distorted multicode DS-CDMA systems with iterative estimation and cancelation of nonlinear distortion [5]. To cope with time-dispersive channels, a linear frequency-domain equalizer (FDE) was employed before the iterative estimation and cancelation of nonlinear distortion effects. However, error propagation effects preclude an efficient estimation and cancelation of nonlinear distortion effects when in the presence of severe nonlinear distortion and/or at moderate and low signal-to-noise ratios (SNR) [5]. When suitable channel coding schemes are employed the working region usually corresponds to low or moderate SNR values, which reduces the interest of those techniques. To avoid noise enhancement effects the FDE is optimized under the minimum mean-squared error (MMSE) criterium [7]. In DS-CDMA this can mean high residual interference levels especially when different powers are assigned to different spreading codes. To improve the performance the linear FDE can be replaced by an iterative block decision feedback equalization (IB-DFE) [8], [9], [10].

In this paper we consider multi-resolution broadcasting systems using CP-assisted DS-CDMA schemes where we have several parallel streams with different powers. The transmitted signals have very low PMEPR thanks to the adoption of iterative $\mathrm{C} \& \mathrm{~F}$ techniques. We modify the approach of [5] so as to cope with its major limitations, namely: the use of a linear FDE; the separate implementation of the FDE part and estimation and compensation of nonlinear distortion effects; error propagation effects in the estimation and compensation of nonlinear distortion; poor performance in the presence of severely nonlinear distortion effects and/or at low-to-moderate SNRs. We consider an IB-DFE receiver with estimation and cancelation of nonlinear distortion effects for each iteration. To improve the performance at low-to-moderate SNR we consider a turbo receiver combined with a threshold-based cancelation of nonlinear distortion effects. We also consider a turbo variant of our receiver where the channel decoding is performed for each iteration.

\section{TRAnsmitter Structure}

In this paper we consider the downlink transmission in DS-CDMA systems employing CP-assisted block transmission techniques combined with FDE schemes. The base station (BS) simultaneously transmits data blocks for $N_{R}$ resolutions. 
For the sake of simplicity, we assume an orthogonal spreading with $K_{r}$ spreading codes associated to the $r$ th resolution and the same spreading factor $K$ for all spreading codes. This means that $\sum_{r=1}^{N_{R}} K_{r} \leq K$. We have a separate channel coding chain for each resolution (channel encoder, interleaver, etc.) as shown in Fig. 1(a). The coded bits associated to the $r$ th resolution are interleaved and mapped in the symbols $\left\{a_{m, r^{\prime}} ; r^{\prime} \in \Psi_{r}\right\}$, with $\Psi_{r}$ denoting a set with the indexes $r^{\prime}$ of the spreading codes associated to the $r$ th resolution (naturally, it is assumed that $\Psi_{r_{1}} \cap \Psi_{r_{2}}=\emptyset$ for $r_{1} \neq r_{2}$, i.e., different spreading codes are assigned to different resolutions). For the sake of simplicity, we assume that all $K$ orthogonal spreading codes are used (the extension to other cases is straightforward), which means that $\bigcup_{r=1}^{N_{R}} \Psi_{r}=\{0,1, \ldots, K-1\}$. The block of chips to be transmitted by the BS is $\left\{s_{n} ; n=0,1, \ldots, N-1\right\}$, where the "overall" chip symbol, $s_{n}$, is given by $s_{n}=$ $\sum_{r=1}^{N_{R}} \sum_{r^{\prime} \in \Psi_{r}} \xi_{r} s_{n, r^{\prime}}$, with $s_{n, r^{\prime}}=c_{n, r^{\prime}} a_{\lfloor n / K\rfloor, r^{\prime}}$ denoting the $n$th chip for the $r^{\prime}$ th spreading code $(\lfloor x\rfloor$ denotes 'larger integer not higher that $\left.x^{\prime}\right)$, where $\left\{c_{n, r^{\prime}} ; n=0,1, \ldots, N-1\right\}$ is the corresponding spreading sequence and $\left\{a_{m, r^{\prime}} ; m=\right.$ $0,1, \ldots, N / K-1\}$ is the block of symbols associated to the $r^{\prime}$ th spreading code. The power assigned to the $r$ th resolution is proportional to $\left|\xi_{r}\right|^{2}$.

To reduce the PMEPR of the transmitted signals we consider the transmitter structure proposed in [11] and depicted in Fig. 1(b). The block of modified samples $\left\{s_{n}^{T x} ; n=\right.$ $0,1, \ldots, N-1\}$ is formed from the original block of samples $\left\{s_{n} ; n=0,1, \ldots, N-1\right\}$ in the following way: The original block of samples is passed to the frequency-domain by a $N$ point discrete Fourier transform (DFT), leading to the block $\left\{S_{k} ; k=0,1, \ldots, N-1\right\}$. Then $N^{\prime}-N$ zeros are added to the block $\left\{S_{k} ; k=0,1 \ldots, N-1\right\}$ so as to form the augmented block $\left\{S_{k}^{\prime} ; k=0,1 \ldots, N^{\prime}-1\right\}$. An inverse discrete Fourier transform (IDFT) brings the augmented block $\left\{S_{k}^{\prime} ; k=0,1 \ldots, N^{\prime}-1\right\}$ back to the time-domain (see Fig. $1(\mathrm{c}))$, resulting the block $\left\{s_{n}^{\prime} ; n=0,1, \ldots, N^{\prime}-1\right\}$. These time-domain samples, which can be regarded as a sampled version of the DS-CDMA block, with the oversampling factor $M_{T x}=N^{\prime} / N$, are submitted to a nonlinear operation so as to reduce the corresponding PMEPR, leading to the modified samples $s_{n}^{C}=g_{C}\left(\left|s_{n}^{\prime}\right|\right) \exp \left(j \arg \left(s_{n}^{\prime}\right)\right)$. A possible nonlinear characteristic is an ideal envelope clipping with clipping level $s_{M}$. A DFT brings the nonlinearly modified samples back to the frequency-domain, leading to the block $\left\{S_{k}^{C} ; k=\right.$ $\left.0,1, \ldots, N^{\prime}-1\right\}$, where a shaping operation corresponding to a frequency-domain filtering is performed so as to obtain the block $\left\{S_{k}^{C F}=S_{k}^{C} G_{k} ; k=0,1, \ldots, N^{\prime}-1\right\}$, with $G_{k}=0$ for the out-of-band samples and 1 for the in-band ones.

To reduce the PMEPR regrowth associated to the filtering operation, the signal processing operations which lead from $\left\{S_{k}^{\prime} ; k=0,1 \ldots, N^{\prime}-1\right\}$ to $\left\{S_{k}^{C F} ; k=0,1 \ldots, N^{\prime}-1\right\}$ in Fig. 1(c) are repeated, in an iterative way, $L$ times leading to the block $\left\{S_{k}^{C F(L)} ; k=0,1, \ldots, N^{\prime}-1\right\}$ which is used to form the "final" frequency-domain block $\left\{S_{k}^{T x} ; k=\right.$ $0,1, \ldots, N-1\}$ by removing $N^{\prime}-N$ zero-valued frequency-

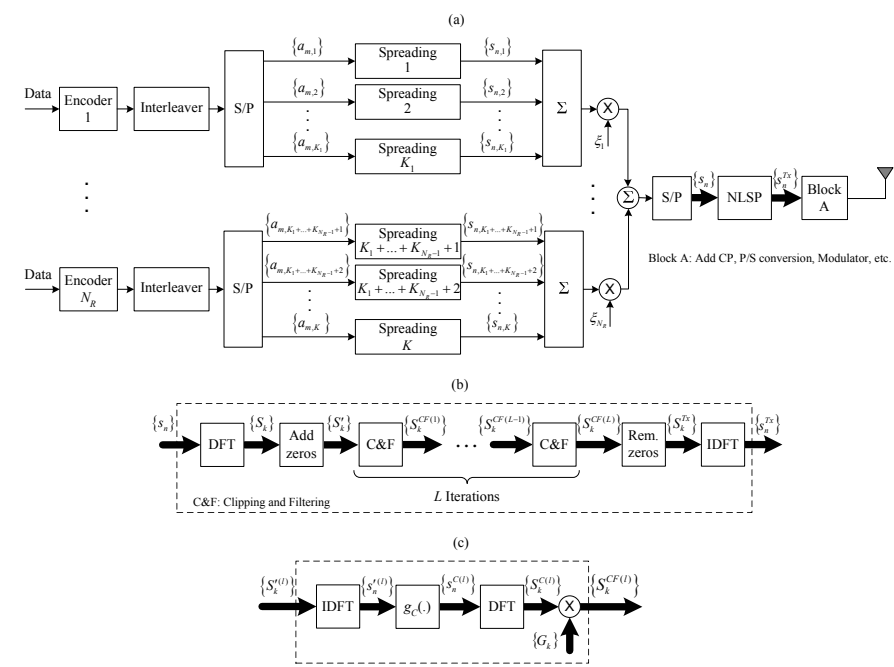

Fig. 1. Transmitter structure considered in this paper (a), detail of the nonlinear signal processing (NLSP) block (b) and clipping and filtering (C\&F) block (c).

domain samples, i.e.,

$$
S_{k}^{T x}=\left\{\begin{array}{ll}
S_{k}^{C F(L)}, & 0 \leq k \leq \frac{N}{2}-1 \\
S_{N^{\prime}-N+k}^{C F(L)}, & \frac{N}{2} \leq k \leq N-1
\end{array} .\right.
$$

Finally, the corresponding IDFT is computed, leading to the block of modified samples $\left\{s_{n}^{T x} ; n=0,1, \ldots, N-1\right\}$. The rest of the transmitter is similar to a conventional, $\mathrm{CP}$-assisted DS-CDMA transmitter (CP insertion, D/A conversion, etc.).

For a given size- $N$ input block with duration $T$, a specific signal processing scheme can be designed through the selection of $M_{T x}=N^{\prime} / N$, the nonlinear device and the number of C\&F iterations. The block $\left\{s_{n}^{\prime} ; n=0,1, \ldots, N^{\prime}-\right.$ 1\} can be regarded as a sampled version of $s(t)=$ $\sum_{n=-\infty}^{+\infty} s_{n}^{\prime} h_{T}\left(t-n \frac{T}{N^{\prime}}\right)$, with the oversampling factor $M_{T x}$, provided that the roll-off factor of the reconstruction filter $h_{T}(t)$ is small. Clearly, the PMEPR of the transmitted signal depends on the adopted pulse shape $h_{T}(t)$. For a square-root raised cosine pulse there is a slight increase in the PMEPR with the roll-off factor (less then $1 \mathrm{~dB}$ [3]).

The nonlinear operation can be selected so as to ensure a PMEPR reduction and the subsequent frequency-domain operation using the set $\left\{G_{k} ; k=0,1 \ldots, N^{\prime}-1\right\}$ provides a complementary filtering effect, eliminating the out-of-band distortion effects of the nonlinearity. However, this filtering operation produces some regrowth on the envelope fluctuations. By repeatedly using, in an iterative way, the nonlinear operation and the subsequent frequency-domain filtering, we can achieve lower envelope fluctuations while preserving a low out-of-band radiation level.

\section{Statistical Characterization of the TRANSMITTED SIGNALS}

In this section we present a statistical characterization of the modified time-domain samples $\left\{s_{n}^{T x} ; n=0,1, \ldots, N-1\right\}$. This characterization is accurate whenever the number of spreading codes is high enough (say, several tens of spreading 
codes) to allow a Gaussian approximation of conventional DS-CDMA signals (to validate the Gaussian approximation the power associated to a given spreading code cannot be a significant fraction of the total power). This statistical characterization can then be used for performance evaluation purposes, as described in the following.

Let us first assume that the signal at the input of the memoryless nonlinear device has a Gaussian nature. In that case, it is well-known that the signal at the nonlinearity output can be decomposed into uncorrelated "useful" and "self-interference" components [3]:

$$
s_{n}^{C}=\alpha s_{n}^{\prime}+d_{n}
$$

where $E\left[s_{n}^{\prime} d_{n^{\prime}}^{*}\right]=0$ and $\alpha=E\left[\left|s_{n}^{\prime}\right| g_{C}\left(\left|s_{n}^{\prime}\right|\right)\right] / E\left[\left|s_{n}^{\prime}\right|^{2}\right]$. Clearly, the average power of the useful component at the nonlinearity output is $P_{N L}^{S}=\left|\alpha^{2}\right| \sigma^{2}$, and the average power of the self-interference component is $P_{N L}^{I}=P_{N L}-P_{N L}^{S}$, where $P_{N L}=E\left[g_{C}^{2}\left(\left|s_{n}^{\prime}\right|\right)\right] / 2$ denotes the average power of the signal at the nonlinearity output.

It can be shown that the autocorrelation of the output samples $R_{s}^{C}\left(n-n^{\prime}\right)=E\left[s_{n}^{C} s_{n^{\prime}}^{C *}\right]$ can be expressed as a function of the autocorrelation of the input samples $R_{s}\left(n-n^{\prime}\right)=E\left[s_{n}^{\prime} s_{n^{\prime}}^{\prime *}\right]$ as follows [3]:

$$
R_{s}^{C}\left(n-n^{\prime}\right)=\sum_{\gamma=0}^{+\infty} 2 P_{2 \gamma+1} \frac{\left[R_{s}\left(n-n^{\prime}\right)\right]^{\gamma+1}\left[R_{s}^{*}\left(n-n^{\prime}\right)\right]^{\gamma}}{\left[R_{s}(0)\right]^{2 \gamma+1}},
$$

where the coefficient $P_{2 \gamma+1}$ denotes the total power associated to the inter-modulation product (IMP) of order $2 \gamma+1$, which can be calculated as described in [3]. If $E\left[s_{n} s_{n^{\prime}}^{*}\right]=2 \sigma_{s}^{2} \delta_{n, n^{\prime}}$ (where $\delta_{n, n^{\prime}}=1$ if $n=n^{\prime}$ and 0 otherwise) then $E\left[S_{k} S_{k^{\prime}}^{*}\right]=$ $2 N \sigma_{s}^{2} \delta_{n, n^{\prime}}$ and

$$
R_{s}\left(n-n^{\prime}\right)=2 \sigma^{2} \frac{\operatorname{sinc}\left[\frac{N\left(n-n^{\prime}\right)}{N^{\prime}}\right]}{\operatorname{sinc}\left(\frac{n-n^{\prime}}{N^{\prime}}\right)} \exp \left[-\frac{j \pi\left(n-n^{\prime}\right)}{N^{\prime}}\right]
$$

$$
\left(n, n^{\prime}=0,1, \ldots, N^{\prime}-1\right) \text {, with } \sigma^{2}=\frac{N^{2}}{\left(N^{\prime}\right)^{2}} \sigma_{s}^{2}=\frac{\sigma_{s}^{2}}{M_{T x}^{2}} \text {. }
$$

Since $R_{s}^{C}\left(n-n^{\prime}\right)=|\alpha|^{2} R_{s}\left(n-n^{\prime}\right)+R_{d}\left(n-n^{\prime}\right)$, where $R_{d}\left(n-n^{\prime}\right)=E\left[d_{n} d_{n^{\prime}}^{*}\right]$, it can be easily recognized that $P_{1}=$ $P_{N L}^{S}=|\alpha|^{2} \sigma^{2}$ and $R_{d}\left(n-n^{\prime}\right)$ is obtained by using $\sum_{\gamma=1}^{\infty}$ instead of $\sum_{\gamma=0}^{\infty}$ in the right-hand side of (3).

Having in mind (2) and the signal processing chain in Fig. 1(c), the frequency-domain block $\left\{S_{k}^{C F}=S_{k}^{C} G_{k} ; k=\right.$ $\left.0,1, \ldots, N^{\prime}-1\right\}$ can obviously be decomposed into useful and self-interference components: $S_{k}^{C F}=\alpha S_{k}^{\prime} G_{k}+D_{k} G_{k}$, where $\left\{D_{k} ; k=0,1, \ldots, N^{\prime}-1\right\}$ denotes the DFT of $\left\{d_{n} ; n=0,1, \ldots, N^{\prime}-1\right\}$. It can be shown that $E\left[D_{k}\right]=0$ and $E\left[D_{k} D_{k^{\prime}}^{*}\right]=N^{\prime} G_{d}(k) \delta_{k, k^{\prime}}\left(k, k^{\prime}=0,1, \ldots, N^{\prime}-1\right)$, where $\left\{G_{d}(k) ; k=0,1, \ldots, N^{\prime}-1\right\}=$ DFT $\left\{R_{d}(n) ; n=\right.$ $\left.0,1, \ldots, N^{\prime}-1\right\}$. Similarly, $E\left[S_{k}^{C} S_{k^{\prime}}^{C *}\right]=N^{\prime} G_{s}^{C}(k) \delta_{k, k^{\prime}}$ where $\left\{G_{s}^{C}(k)=|\alpha|^{2} G_{s}(k)+G_{d}(k) ; k=0,1, \ldots, N^{\prime}-\right.$ $1\}=\operatorname{DFT}\left\{R_{s}^{C}(n) ; k=0,1, \ldots, N^{\prime}-1\right\}$, with $R_{s}^{C}(n)$ given by (3), and $\left\{G_{s}(k) ; k=0,1, \ldots, N^{\prime}-1\right\}=$ DFT $\left\{R_{s}(n) ; n=0,1, \ldots, N^{\prime}-1\right\}$. Therefore, $E\left[S_{k}^{C F} S_{k^{\prime}}^{C F *}\right]=$ $G_{k} G_{k^{\prime}}^{*} E\left[S_{k}^{C} S_{k^{\prime}}^{C *}\right]=N^{\prime}\left|G_{k}\right|^{2} G_{s}^{C}(k) \delta_{k, k^{\prime}}$.
The "final" frequency-domain block can also be decomposed into uncorrelated useful and self-interference terms, $S_{k}^{T x}=\alpha S_{k}+D_{k}^{T x}$, with

$$
D_{k}^{T x}=\left\{\begin{array}{ll}
D_{k}, & 0 \leq k \leq \frac{N}{2}-1 \\
D_{N^{\prime}-N+k}, & \frac{N}{2} \leq k \leq N-1
\end{array} .\right.
$$

This means that $s_{n}^{T x}=\alpha s_{n}+d_{n}^{T x}$, where $\left\{d_{n}^{T x} ; n=\right.$ $0,1, \ldots, N-1\}$ is the IDFT of $\left\{D_{k}^{T x} ; k=0,1, \ldots, N-1\right\}$.

For the iterative signal processing scheme in Fig. 1(b) the Gaussian approximation for the samples at the input to the nonlinearity is no longer valid after the first iteration. Therefore, the method for modeling the transmitted blocks needs to be modified. Our simulations have shown that

$$
S_{k}^{C F(l)} \approx \alpha_{k}^{(l)} S_{k}^{\prime} G_{k}+D_{k}^{(l)} G_{k},
$$

with $\alpha_{k}^{(l)}$ depending on $k$ when $l>1$. This means that the $k$ th component of the frequency-domain block, for the lth iteration, can still be decomposed as a sum of two uncorrelated components. The statistical characterization concerning the first iteration, described above, can be regarded as a special case of (6) with constant $\alpha_{k}^{(1)}$; in this case, the values of $\alpha_{k}^{(1)}$ and $E\left[\left|D_{k}^{(1)}\right|^{2}\right]$ can be obtained analytically as described in the previous subsection. For the remaining iterations $(l>1)$ the values of $\alpha_{k}^{(l)}$ and $E\left[\left|D_{k}^{(l)}\right|^{2}\right]$ can be obtained by simulation in the following way: $\alpha_{k}^{(l)}=E\left[S_{k}^{C(l)} S_{k}^{* *}\right] / E\left[\left|S_{k}^{\prime}\right|^{2}\right]$ and $E\left[\left|D_{k}^{(l)}\right|^{2}\right]=E\left[\left|S_{k}^{C(l)}-\alpha_{k}^{(l)} S_{k}^{\prime}\right|^{2}\right]$, respectively. Our simulations also indicate that $E\left[D_{k}^{(l)}\right]=0$ and $E\left[D_{k}^{(l)} D_{k^{\prime}}^{(l) *}\right] \approx$ $0, k \neq k^{\prime}$, as with the basic transmitter structure (i.e., with a single $\mathrm{C} \& \mathrm{~F}$ procedure).

From (1) and (6), it is clear that the samples $\left\{S_{k}^{T x} ; k=\right.$ $0,1, \ldots, N-1\}$ can be decomposed into uncorrelated "useful" and "self-interference" terms, i.e., $S_{k}^{T x}=\alpha_{k}^{T x} S_{k}+D_{k}^{T x}$, with $\alpha_{k}^{T x}=\alpha_{k}^{(L)}$ for $0 \leq k \leq \frac{N}{2}-1$ and $\alpha_{N^{\prime}-N+k}^{(L)}$ for $\frac{N}{2} \leq k \leq N-1 .\left\{D_{k}^{T x} ; k=0,1, \ldots, N-1\right\}$ is related to $\left\{D_{k}^{(L)} ; k=0,1, \ldots, N^{\prime}-1\right\}$ as in (5). This means that $s_{n}^{T x}=s_{n}^{U}+d_{n}^{T x}$, where $\left\{s_{n}^{U} ; n=0,1, \ldots, N-1\right\}$ is the IDFT of $\left\{\alpha_{k}^{T x} S_{k} ; k=0,1, \ldots, N-1\right\}$ (corresponding to the "useful" component of the transmitted signal) and $\left\{d_{n}^{T x} ; n=\right.$ $0,1, \ldots, N-1\}$ is the IDFT of $\left\{D_{k}^{T x} ; k=0,1, \ldots, N-1\right\}$ (corresponding to the "self-interference" component on the transmitted signal). Therefore, the modified samples $\left\{s_{n}^{T x} ; n=\right.$ $0,1, \ldots, N-1\}$ can always be decomposed into uncorrelated "useful" and "self-interference" components regardless of the number of C\&F procedures. However, after the first iteration, the "useful" component is no longer proportional to the original samples, $\left\{s_{n} ; n=0,1, \ldots, N-1\right\}$, due to the filtering effect inherent to $\alpha_{k}^{T x}$.

\section{ReCEIVER DESIGN}

\section{A. Receiver Structure}

Since the orthogonality between spreading codes is lost in a time-dispersive channel, we perform an FDE before the "despreading" procedure [9]: after removing the $\mathrm{CP}$, the 
received time-domain block $\left\{y_{n} ; n=0,1, \ldots, N-1\right\}$ is passed to the frequency-domain by a DFT leading to the block $\left\{Y_{k}=H_{k} S_{k}^{T x}+N_{k} ; k=0,1, \ldots, N-1\right\}$, with $H_{k}$ and $N_{k}$ denoting the channel frequency response and the noise term for the $k$ th frequency, respectively (as usually, it is assumed that the CP is longer than the overall channel impulse response).

Since $S_{k}^{T x}=\alpha_{k}^{T x} S_{k}+D_{k}^{T x}$, it can be shown that the optimum FDE coefficients in the MMSE sense are given by

$$
F_{k}=\frac{\alpha_{k}^{T x *} E\left[\left|S_{k}\right|^{2}\right] H_{k}^{*}}{\left\{E\left[\left|\alpha_{k}^{T x} S_{k}\right|^{2}\right]+E\left[\left|D_{k}^{T x}\right|^{2}\right]\right\}\left|H_{k}\right|^{2}+E\left[\left|N_{k}\right|^{2}\right]},
$$

where $E\left[\left|D_{k}^{T x}\right|^{2}\right]$ can be obtained as described in Sec. III. The frequency-domain block at the output of the FDE is then $\left\{\tilde{S}_{k}=Y_{k} F_{k} ; k=0,1, \ldots, N-1\right\}$.

The data block associated with the $r^{\prime}$ th spreading codes could be estimated by despreading the time-domain block at the FDE output, $\left\{\tilde{s}_{n} ; n=0,1, \ldots, N-\right.$ $1\}=\operatorname{IDFT}\left\{\tilde{S}_{k} ; k=0,1, \ldots, N-1\right\}$, i.e., from the samples $\tilde{a}_{m, r^{\prime}}=\sum_{n^{\prime}=n K}^{n K+K} \tilde{s}_{n^{\prime}} c_{n^{\prime}, r^{\prime}}^{*}$.

Clearly, the nonlinear effects lead to some BER degradation relatively to conventional DS-CDMA schemes, especially when a low PMEPR is intended and/or for codes with small assigned power. This degradation results from both the "useless" transmitted power spent on self-interference and the received self-interference being added to the channel noise.

To improve performance we consider the iterative receiver for CP-assisted multicode DS-CDMA depicted in Fig. 2. This receiver employs an IB-DFE with soft decisions [12] (instead of the linear FDE) which is combined with estimation and compensation of nonlinear self-interference components. The receiver can be described as follows: For a given iteration, the signal at the output of the FDE is $\tilde{S}_{k}=F_{k}\left(Y_{k}-\right.$ $\left.H_{k} \hat{D}_{k}^{T x}\right)-B_{k} \bar{S}_{k}$, where $\left\{B_{k} ; k=0,1, \ldots, N-1\right\}$ is the block of feedback coefficients, $\left\{\bar{S}_{k} ; k=0,1, \ldots, N-1\right\}$ are the average values of $\left\{S_{k} ; k=0,1, \ldots, N-1\right\}$ associated to the previous iteration, conditioned to the FDE output, and $\left\{\hat{D}_{k}^{T x} ; k=0,1, \ldots, N-1\right\}$ are estimates of the transmitted nonlinear self-interference components obtained by submitting $\bar{S}_{k}$ to a replica of nonlinear device at the transmitter (see Fig. 2) (it is assumed that the nonlinear characteristic $g_{C}\left(\left|s_{n}^{\prime}\right|\right)$ adopted at the transmitter is known at the receiver). After the despreading operation, the data estimates for each spreading code $\left\{\bar{a}_{m, r^{\prime}}\right\}$ are obtained by submitting $\left\{\tilde{a}_{m, r^{\prime}}\right\}$ to a softdecision device and used to form the estimates of the chip samples $\left\{\bar{s}_{n} ; n=0,1, \ldots, N-1\right\}$.

We can also define a receiver that, as turbo equalizers, employs the channel decoder outputs instead of the uncoded soft decisions in the feedback loop of the IB-DFE. The receiver structure, that will be denoted as Turbo-FDE, is similar to the IB-DFE with soft decisions, but with soft-in-soft-out (SISO) channel decoder outputs employed in the feedback loop. The SISO block, that can be implemented as defined in [13], provides the log-likelihood ratios (LLR) of both the "information bits" and the "coded bits". The input of the SISO block are LLRs of the "coded bits" from the IB-DFE, after

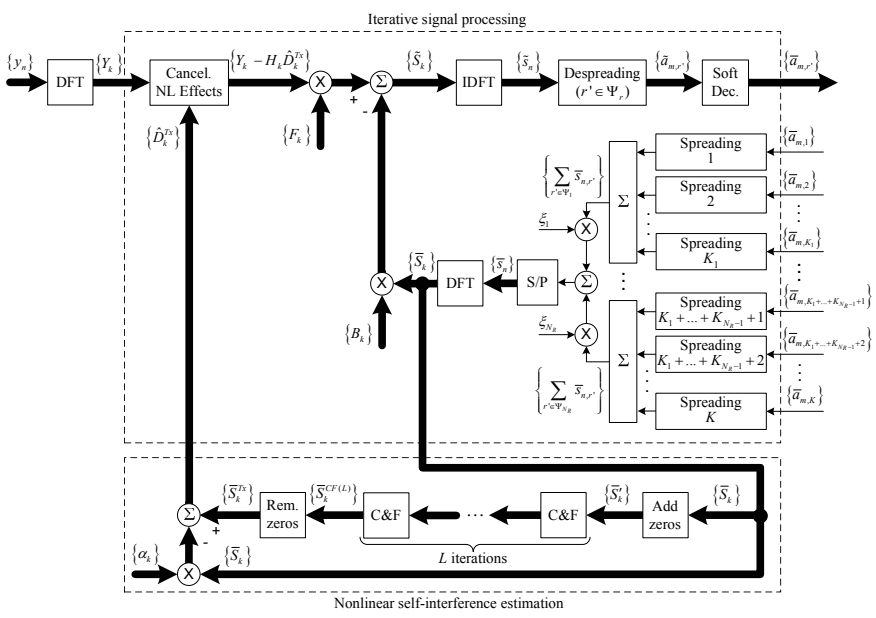

Fig. 2. Iterative receiver structure with estimation and compensation of nonlinear self-interference effects.

being appropriately deinterleaved ${ }^{2}$.

\section{B. Computation of Receiver Parameters}

The optimum values of the feedforward and feedback coefficients, in the MMSE sense, are

$$
F_{k}=\frac{\kappa \alpha_{k}^{T x *} H_{k}^{*}}{\beta+\eta_{k}\left|H_{k}\right|^{2}+\left(1-\rho^{2}\right)\left|\alpha_{k}^{T x} H_{k}\right|^{2}}
$$

and $B_{k}=F_{k} H_{k} \alpha_{k}^{T x}-1$, respectively, where $\kappa$ is selected to ensure that $\sum_{k=0}^{N-1} F_{k} H_{k} \alpha_{k}^{T x} / N=1, \beta=$ $E\left[\left|N_{k}\right|^{2}\right] / E\left[\left|S_{k}\right|^{2}\right], \quad \eta_{k}=E\left[\left|D_{k}^{T x}-\hat{D}_{k}^{T x}\right|^{2}\right] / E\left[\left|S_{k}\right|^{2}\right]$, and the correlation coefficient $\rho$ can be regarded as a measure of the reliability of the decisions used in the feedback loop, from the previous iteration, given by $\rho=\left(\sum_{r=1}^{N_{R}} \xi_{r}^{2} \sum_{r^{\prime} \in \Psi_{r}} \rho_{r^{\prime}}\right) /\left(\sum_{r=1}^{N_{R}} \xi_{r}^{2} K_{r}\right)$ with $\rho_{r^{\prime}}=$ $\frac{K}{2 N} \sum_{m=0}^{\frac{N}{K}-1}\left(\left|\operatorname{Re}\left\{\bar{a}_{m, r^{\prime}}\right\}\right|+\left|\operatorname{Im}\left\{\bar{a}_{m, r^{\prime}}\right\}\right|\right)$.

It can be shown that the "overall chip averages" are

$$
\bar{s}_{n}=\sum_{r=1}^{N_{R}} \xi_{r} \sum_{r^{\prime} \in \Psi_{r}} c_{n, r^{\prime}} \bar{a}_{\lfloor n / K\rfloor, r^{\prime}} .
$$

For a QPSK constellation, the average data values are

$$
\bar{a}_{m, r^{\prime}}=\tanh \left(\frac{\operatorname{Re}\left\{\tilde{a}_{m, r^{\prime}}\right\}}{\sigma_{r^{\prime}}^{2}}\right)+\tanh \left(\frac{\operatorname{Im}\left\{\tilde{a}_{m, r^{\prime}}\right\}}{\sigma_{r^{\prime}}^{2}}\right),
$$

where $\tilde{a}_{m, r^{\prime}}$ denotes the despreaded symbols and

$$
\begin{aligned}
\sigma_{r^{\prime}}^{2} & =\frac{1}{2} E\left[\left|a_{m, r^{\prime}}-\tilde{a}_{m, r^{\prime}}\right|^{2}\right] \\
& \approx \frac{K}{2 N} \sum_{m=0}^{N} E\left[\left|\hat{a}_{m, r^{\prime}}-\tilde{a}_{m, r^{\prime}}\right|^{2}\right],
\end{aligned}
$$

with $\hat{a}_{m, r^{\prime}}$ denoting the hard decisions associated to $\tilde{a}_{m, r^{\prime}}$.

With respect to $\eta_{k}$, for the first iteration $\hat{D}_{k}^{T x}=0$ and we can use the method of [4] to obtain $E\left[\left|D_{k}^{T x}\right|^{2} \mid\right.$. For the remaining iterations it has to be obtained by simulation, as described in the following subsection.

\footnotetext{
${ }^{2}$ As usual, it is assumed that the bits at the channel encoder output are interleaved before being mapped into the adopted constellation.
} 


\section{Computation of Nonlinear Distortion Estimates}

An important issue in our receiver is the estimation of nonlinear distortion effects. One possibility is to submit the soft-decision chip estimates $\left\{\bar{s}_{n} ; n=0,1, \ldots, N-1\right\}$ to a replica of the nonlinear signal processing chain at the transmitter so as to obtain the self-interference estimates

$$
\left.\hat{D}_{k}^{T x}\right|_{\left\{\bar{s}_{n}\right\}}=\left.\bar{S}_{k}^{T x}\right|_{\left\{\bar{s}_{n}\right\}}-\alpha_{k}^{T x} \bar{S}_{k},
$$

where $\left\{\bar{S}_{k} ; k=0,1, \ldots, N-1\right\}$ is the DFT of $\left\{\bar{s}_{n} ; n=\right.$ $0,1, \ldots, N-1\}$ (as shown in Fig. 2).

As an alternative, we could also obtain the nonlinear distortion estimates by submitting the hard decisions $\left\{\hat{s}_{n} ; n=\right.$ $0,1, \ldots, N-1\}$ to the nonlinear signal processing chain instead of $\left\{\bar{s}_{n} ; n=0,1, \ldots, N-1\right\}$, i.e.,

$$
\left.\hat{D}_{k}^{T x}\right|_{\left\{\hat{s}_{n}\right\}}=\left.\hat{S}_{k}^{T x}\right|_{\left\{\hat{s}_{n}\right\}}-\alpha_{k}^{T x} \hat{S}_{k},
$$

where $\left\{\hat{S}_{k} ; k=0,1, \ldots, N-1\right\}$ is the DFT of $\left\{\hat{s}_{n} ; n=\right.$ $0,1, \ldots, N-1\}$. To avoid error propagation, these estimates could be weighted by the correlation coefficient $\rho$, which can be regarded as the overall reliability of the decisions used in the feedback loop. This leads to the estimates

$$
\hat{D}_{k}^{T x}=\left.\rho \hat{D}_{k}^{T x}\right|_{\left\{\hat{s}_{n}\right\}} .
$$

However, our simulations results showed that for small values of $\rho$, using these estimates to remove nonlinear distortion effects can be worse than not doing it, since $E\left[\left|D_{k}^{R e s}\right|^{2}\right]>$ $E\left[\left|D_{k}^{T x}\right|^{2}\right]$ where $D_{k}^{R e s}=D_{k}^{T x}-\hat{D}_{k}^{T x}$ denotes the residual nonlinear self-interference. Therefore, the compensation should only take place when the residual nonlinear selfinterference is smaller than the nonlinear self-interference in the transmitted signals and when the reliability of the "overall chip" estimates is above a given threshold. We will assume that

$$
\begin{aligned}
E\left[\left|D_{k}^{R e s}\right|^{2}\right] & \approx f(\rho) E\left[\left|D_{k}^{T x}\right|^{2}\right] \\
& =\left(a_{1} \rho^{2}+a_{2} \rho+a_{3}\right) E\left[\left|D_{k}^{T x}\right|^{2}\right]
\end{aligned}
$$

where $a_{1}, a_{2}$ and $a_{3}$ are coefficients that depend on the adopted normalized clipping level $s_{M} / \sigma$. Approximation (15) is used to compute $\eta_{k}$ and as a threshold to trigger the compensation of nonlinear distortion effects.

\section{Performance Results}

In this section we present a set of performance results concerning our improved receivers for multi-resolution broadcasting in DS-CDMA systems employing CP-assisted block transmission techniques, combined with FDE schemes, where an iterative cancelation of deliberate nonlinear distortion effects is carried out. Unless otherwise stated, the transmitter (i.e., the BS) simultaneously transmits data blocks for $N_{R}=4$ resolutions. The coded bits associated to each resolution are interleaved before being mapped into QPSK symbols under a Gray mapping rule (a bit-level random interleaving is performed over 5 fast Fourier transform (FFT) blocks). We consider an orthogonal spreading with $K_{r}=64, r=1, \ldots, 4$, spreading codes associated to each resolution and the same spreading factor $K=N=256$ for all spreading codes (this corresponds to a fully loaded system). To reduce the PMEPR of the transmitted signals while maintaining the spectral occupations of conventional DS-CDMA signals, the BS performs an ideal envelope clipping operation, with normalized clipping level $s_{M} / \sigma$ and an oversampling factor $M_{T x}=N^{\prime} / N=2$, combined with a frequency-domain filtering operation, which can be jointly repeated several times. The power amplifier at the transmitter is quasi-linear within the (reduced) range of variations of the input signal envelope. The receiver (i.e., the MT) knows the characteristic of the PMEPR-reducing signal processing technique employed by the transmitter. We consider a severely time-dispersive channel characterized by the power delay profile type $\mathrm{C}$ for the HIPERLAN/2 (HIgh PERformance Local Area Network) [14], with uncorrelated Rayleigh fading on the different paths and the duration of the $\mathrm{CP}$ is $1 / 5$ of the duration of the useful part of the block (similar results were obtained for other severely time-dispersive channels). Perfect synchronization and channel estimation are assumed at the receiver.

We consider uncoded and coded BER performances with the well-known rate-1/2, 64-state convolutional code with generators $1+D^{2}+D^{3}+D^{5}+D^{6}$ and $1+D+D^{2}+D^{3}+D^{6}$. The SISO decoder is implemented using the Max-Log-MAP approach. As mentioned before, we will denote the receiver that employs uncoded soft decisions in the feedback loop as IB-DFE and the receiver with soft decisions from the SISO channel decoder outputs in the feedback loop as Turbo-FDE.

Let us consider a multi-resolution scenario with $\xi_{1}=$ $1, \xi_{2}=1 / 2, \xi_{3}=1 / 4$ and $\xi_{4}=1 / 8$ (i.e., the power assigned to the $r$ th resolution is $6 \mathrm{~dB}$ below the power assigned to the $(r-1)$ th resolution), assuming a nonlinear transmitter with $s_{M} / \sigma=0.5$ and only one C\&F operation, allowing PMEPR values of about $4.1 \mathrm{~dB}$ (for conventional DS-CDMA schemes with a large number of spreading codes PMEPR $\approx 8.4 \mathrm{~dB}$ ). Figs. 3 and 4 concern the same severely time-dispersive channel mentioned above and show the uncoded BER performance for the IB-DFE receiver and the coded BER performance for both IB-DFE and Turbo-FDE receivers, respectively, for iterations 1 and 4 (naturally, the first iteration corresponds to a linear receiver). For the sake of comparisons, Fig. 3 also includes the performance for a linear transmitter. Clearly, the performance degradation due to the nonlinear distortion effects can be very high, especially for low-power resolutions. We can also observe that the Turbo-FDE receiver significantly outperforms the IB-DFE.

Let us consider a case where we want a transmission with a very low-PMEPR of the DS-CDMA signals, not only by assuming a very low clipping level, but also by repeating several times the $\mathrm{C} \& \mathrm{~F}$ operations to further reduce the PMEPR of the transmitted signals while maintaining the spectral occupation of conventional DS-CDMA schemes. Fig. 5 shows the average coded BER performance for iterations 1 and 4 for Turbo-FDE receiver with $1,2,4$ or $8 \mathrm{C} \& \mathrm{~F}$ 


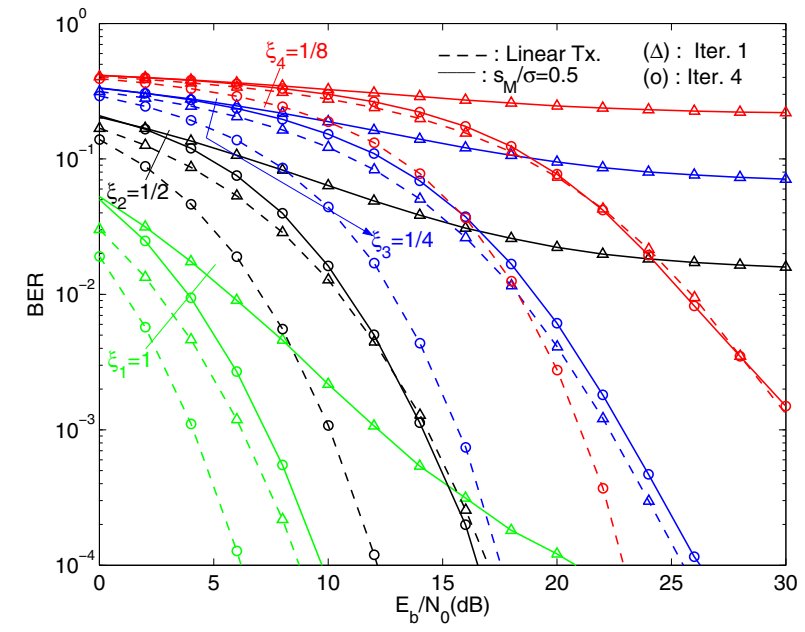

Fig. 3. Uncoded BER performance for iterations 1 and 4 , when $\xi_{1}=$ $1, \xi_{2}=1 / 2, \xi_{3}=1 / 4$ and $\xi_{4}=1 / 8$, for IB-DFE receiver, when linear and nonlinear transmitters with normalized clipping level of $s_{M} / \sigma=0.5$ are considered.

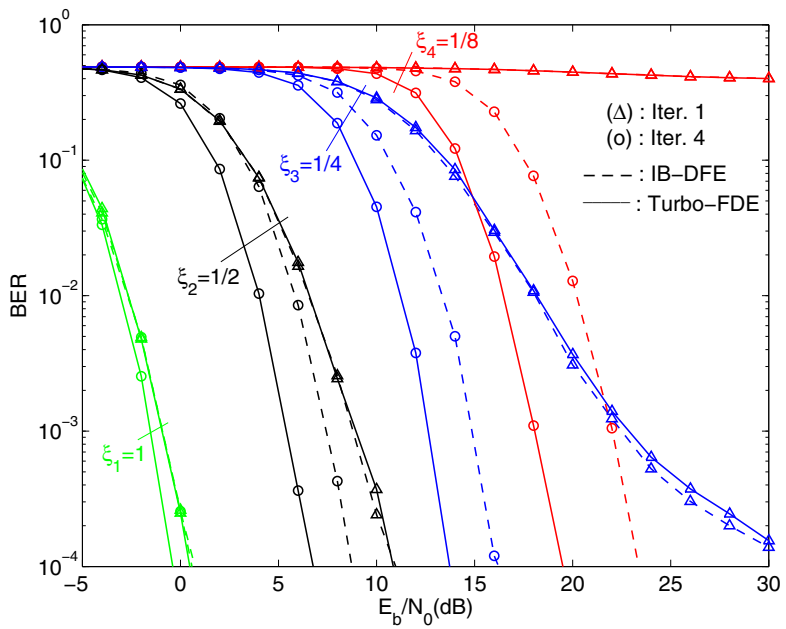

Fig. 4. Coded BER performance for the IB-DFE and Turbo-FDE receivers, for the same scenario of Fig. 3.

iterations at the transmitter and a normalized clipping level of $s_{M} / \sigma=0.5$ (the corresponding PMEPR values are 4.1, 3.0, 2.0 and $1.7 \mathrm{~dB}$, respectively). From this figure it is clear that the performance degradation associated to several $\mathrm{C} \& \mathrm{~F}$ operations is relatively small when Turbo-FDE receivers with estimation and cancelation of nonlinear distortion effects are employed, in spite of the considerable PMEPR reduction of the transmitted signals.

\section{Conclusions and Final Remarks}

In this paper we considered DS-CDMA systems employing iterative clipping and filtering techniques. We presented an improved receiver able to perform an iterative estimation and cancelation of deliberate nonlinear distortion effects. Our performance results show that we can improve significantly the performance with just a few iterations, even with strong nonlinear effects.

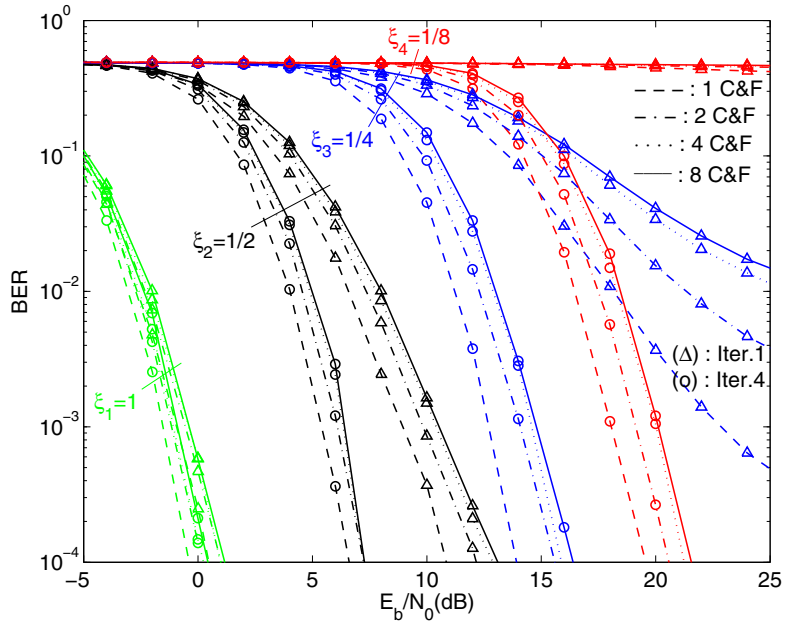

Fig. 5. Coded BER performance for iterations 1 and 4 , when $\xi_{1}=1, \xi_{2}=$ $1 / 2, \xi_{3}=1 / 4$ and $\xi_{4}=1 / 8$, for Turbo-FDE receiver, with $1,2,4$ or $8 \mathrm{C} \& \mathrm{~F}$ iterations at the transmitter and a normalized clipping level of $s_{M} / \sigma=0.5$.

\section{REFERENCES}

[1] N. Guo and L. Milstein, "Uplink Performance Evaluation of Multicode DS/CDMA Systems in the Presence of Nonlinear Distortions", IEEE J. on Sel. Areas in Comm., Vol. 18, No. 8, pp. 1418-1428, Aug. 2000.

[2] O. Väänänen, J. Vankka, T. Viero and K. Halonen, "Effect of Clipping in Wideband CDMA System and Simple Algorithm for Peak Windowing", World Wireless Congress, pp. 614-619. San Francisco, May, 2002.

[3] R. Dinis and A. Palhau, "A Class of Signal-Processing Schemes for Reducing the Envelope Fluctuations of CDMA Signals", IEEE Trans. on Comm., Vol. 53, pp. 882-889, May 2005.

[4] R. Dinis and A. Palhau "A New Approach for Very Low-PMEPR Transmission in the Downlink of a DS/CDMA System", IEEE VTC'04 (Spring), Milan, Italy, May 2004.

[5] R. Dinis and P. Silva, "An Iterative Detection Technique for DS-CDMA Signals with Strong Nonlinear Distortion Effects", IEEE VTC'06 (Fall), pp. 1-5, Montreal, Canada, Sep. 2006.

[6] T. Cover, "Broadcast Channels", IEEE Trans. on Inform. Theory, Vol. 18, No. 1, pp. 2-14, Jan. 1972.

[7] A. Gusmão, R. Dinis and N. Esteves, "On Frequency-domain Equalization and Diversity Combining for Broadband Wireless Communications", IEEE Trans. on Comm., Vol. 51, No. 7, pp. 1029-1033, July 2003.

[8] N. Benvenuto and S. Tomasin, "Block Iterative DFE for Single Carrier Modulation", IEE Elec. Let., Vol. 39, No. 19, pp. 1144-1145, Sep. 2002.

[9] P. Silva and R. Dinis, "An Iterative Frequency-Domain Decision Feedback Receiver for CDMA Systems", IEEE ISWCS'04, pp. 6-10, Mauritius, Sep. 2004.

[10] R. Dinis, P. Silva and A. Gusmão, "IB-DFE Receivers with Space Diversity for CP-Assisted DS-CDMA and MC-CDMA Systems", European Trans. on Telecomm., Vol. 18, No. 7, pp. 791-802, Nov. 2007.

[11] R. Dinis and A. Gusmão, "A Class of Nonlinear Signal Processing Schemes for Bandwidth-Efficient OFDM Transmission with Low Envelope Fluctuation", IEEE Trans. on Comm., Vol. 52, No. 11, pp. 20092018, Nov. 2004.

[12] A. Gusmão, P. Torres, R. Dinis and N. Esteves, "A Turbo FDE Technique for Reduced-CP SC-Based Block Transmission Systems", IEEE Trans. on Comm., Vol. 55, No. 1, pp. 16-20, Jan. 2007.

[13] B. Vucetic and J. Yuan, Turbo Codes: Principles and Applications, Kluwer Academic Publ., 2002.

[14] ETSI, "Channel models for HIPERLAN/2 in Different Indoor Scenarios", ETSI EP BRAN 3ERIO85B, pp. 1-8, March 1998. 